\title{
Respiratory therapy in COVID-19: Which model?
}

\author{
Amit Jain ${ }^{1}$ and D. John Doyle ${ }^{1}$ \\ ${ }^{1}$ Cleveland Clinic Abu Dhabi
}

May 20, 2020

\begin{abstract}
As we deal with the COVID-19 pandemic we face a controversy concerning its pathophysiology and how to integrate available knowledge into practice while awaiting study outcomes. COVID-19's pathophysiology remains elusive, as reflected in putative mechanisms that remain unsupported by robust evidence. Some models draw on clinical observations without reference to supporting data from genomic, proteomic, molecular, physiological, and other data pertaining to human coronaviruses. Consequently, some proposed models for COVID-19 pathophysiology and their corresponding treatment options remain highly divergent. To provide a pathophysiological model that better describes the different phenotypic presentations of the disease in concordance with existing research on the renin-angiotensin system, previously described pathophysiological processes for other human coronavirus infections and the genomic similarities between the SARS and SARS-CoV-2 viruses, we developed a conceptual model, "epithelial-endothelial crosstalk at alveolar-capillary membrane" that we believe can help explain the pathogenesis of COVID-19.
\end{abstract}

\section{Respiratory therapy in COVID-19: Which model?}

\section{Author Information:}

Amit Jain M.D., Anesthesiology Institute, Cleveland Clinic Abu Dhabi, United Arab Emirates

D. John Doyle, M.D., Ph.D., Anesthesiology Institute, Cleveland Clinic Abu Dhabi, United Arab Emirates

\section{Corresponding Author:}

Amit Jain

Anesthesiology Institute, Cleveland Clinic Abu Dhabi, Al Maryah Island, Abu Dhabi

United Arab Emirates

M: +971544103790

Email:amitvasujain@gmail.com

Word and Element Counts:

Word Count: 922

Number of references: 8

Number of figures: 1

Funding Statement: Support was provided solely from institutional and/or departmental sources

Conflicts of Interest: The authors declare no competing interests

Dear Editor 
As we deal with the COVID-19 pandemic we face a controversy concerning its pathophysiology and how to integrate available knowledge into practice while awaiting study outcomes. COVID-19's pathophysiology remains elusive, as reflected in putative mechanisms that remain unsupported by robust evidence. Some models draw on clinical observations without reference to supporting data from genomic, proteomic, molecular, physiological, and other data pertaining to human coronaviruses. Consequently, some proposed models for COVID-19 pathophysiology and their corresponding treatment options remain highly divergent.

A key example is the debate over whether COVID-19 pneumonia presents with typical ARDS features. Since COVID-19 pneumonia patients frequently have hypoxemia that fulfils the Berlin definition for ARDS early in the disease, these patients are often ventilated using high PEEP-low tidal volume strategies, with early intubation being recommended to reduce aerosol-based viral spread. However, of necessity, both these recommendations were based purely on clinical judgment without reference to the underlying pathophysiology (which was lacking at the time of these recommendations) or the outcomes of clinical trials (which were just being launched). Consequently, such recommendations sometimes caused more harm than benefit.

As discussed below, various theories have been proposed but none explain the disease pathophysiology in a satisfactory manner.

1. The Gattinoni ModelGattinoni et al. suggested two phenotypes for COVID-19 pneumonia: "TypeL" and "Type-H" 1 , where patients present initially with the "L-phenotype" characterized by severe hypoxia despite normal lung compliance and low recruitability. Patient self-inflicted lung injury (PSILI) and high PEEP injury is said to cause progression to the "H-phenotype", similar in pathology and treatment to ARDS. They suggested that high PEEP / low tidal volume ventilation protocols are ineffective in Type-L patients and could even cause harm. After noting that the ratio of shunt fraction to fraction of gasless tissue on CT scan averaged $3.0 \pm 2$, suggesting hyperperfusion of gasless tissue 2 , the authors additionally hypothesized that loss of lung perfusion regulation and vasoplegia caused the hypoxia in Type-L patients. 1 Gattinoni et al. later suggested endothelial dysfunction as the reason for pulmonary vasoplegia in Type-L pneumonia3, although no specific mechanism was proposed. Still, the theory received wide recognition and become a basis for ventilatory management in many ICUs worldwide.

2. High altitude pulmonary edema (HAPE)Some physicians compare COVID-19 symptoms to those found in HAPE, offering pulmonary vasoconstriction in similarity to HAPE as the underlying mechanism for hypoxia in Type-L patients. 4 Even though this hypothesis is unsupported by evidence, the authors recommended acetazolamide, nifedipine and phosphodiesterase inhibitors as possible treatment options. Understandably, clinicians with experience with both HAPE and COVID-19 have pushed back on this observation and have argued that the comparison is risky. 5

To provide a pathophysiological model that better describes the different phenotypic presentations of the disease in concordance with existing research on the renin-angiotensin system, previously described pathophysiological processes for other human coronavirus infections and the genomic similarities between the SARS and SARS-CoV-2 viruses, we developed a conceptual model that we believe can help explain the pathogenesis of COVID-19.

In our view the L-phenotype is actually a Stage 2b/Stage 3 manifestation of COVID-19 pneumonia 6 and we offer an 'epithelial-endothelial crosstalk' hypothesis involving alterations in local RAS system as a mechanistic explanation:

1. SARS-CoV-2 enters type-2 pneumocytes following binding to ACE2 receptors, causing downregulation of ACE2 on the alveolar-capillary membrane (ACM). This reduces the level of protective, vasodilatory, anti-proliferation and anti-inflammatory ACE2-Ang 1-7-masR activity and increases the level of vasoconstriction, proliferation, inflammation and pro-fibrotic ACE 1-Ang II-AT1-R activity.

2. Angiotensin II mediated activation of alveolar endothelium rich in AT1 receptors increases the release of endothelin-1 (ET1) and reactive oxygen species (ROS). Meanwhile, ACE2-Ang 1-7-masR mediated constitutive endothelial nitric oxide synthase (eNOS) activation and NO release is relatively inhibited 
(Figure 1). Of note, SARS-CoV2 seems to produce little cytopathic changes in pulmonary epithelium. 7

3. Intense ROS-mediated and ET1-mediated pulmonary vasoconstriction is hypothesized to be the principal mechanism for the initial hypoxia. This vasoconstriction is severe but, uneven, and as a result, capillary beds with relatively less vasoconstriction are disproportionately exposed to elevated microvascular pressures, resulting in recruitment and regional over-perfusion. This results in increased shunt fraction and hypoxia. As the disruption of the ACM progresses, proteins, fibrin, cells, and fluid leak into the alveolar space, resulting in bilateral patchy ground glass opacities on CT scan. Additionally, the finding of elevated pulmonary artery pressure, an enlarged right-atrium, and diminished right-ventricle (RV) function in patients with $\mathrm{P} / \mathrm{F}$ ratios [?] $150 \mathrm{mmHg} 8$ suggest pulmonary vasoconstriction as a mechanism for RV longitudinal strain (RVLS). Notably, RVLS has been linked with increased risk for progression to ARDS and mortality in COVID-19.

4. Following ACM disruption, SARS CoV-2 enters the pulmonary capillaries and infects the pulmonary endothelial cells via ACE-2 protein on the luminal surfaces. As a result, endothelial cells assume a 'proinflammatory'/ 'procoagulant' phenotype, causing thrombotic occlusions of heterogeneous regions of pulmonary vasculature.

5. Activated endothelial cells accelerate apoptosis of alveolar epithelial and endothelial cells and produce a cytokine storm syndrome (CSS).

6. Hematogenous spread resulting in direct vial-induced injury and CSS can explain extrapulmonary disease manifestations.

In sum, our hypothesis supports the observations of Solaimanzadeh and Gattinoni et al. but suggest an alternate pathophysiology pathway common to SARS and MERS. In our opinion, the stratification of COVID-19 patients using the Berlin criteria is problematic. Finally, our hypothesis has treatment implications warranting investigation. Ventilation strategies as proposed by Marini et al.3 may be effective but early intubation may not prevent a transition to ARDS. An RV protective approach including early prone positioning and inhaled NO, as well as steroids and anticoagulation may prove useful.

\section{References}

1. Gattinoni L, Chiumello D, Caironi P, et al. COVID-19 pneumonia: different respiratory treatments for different phenotypes? Intensive Care Med . 2020. doi:10.1007/s00134-020-06033-2

2. Gattinoni L, Coppola S, Cressoni M, Busana M, Rossi S, Chiumello D. Covid-19 Does Not Lead to a "Typical" Acute Respiratory Distress Syndrome. Am J Respir Crit Care Med . 2020. doi:10.1164/rccm.202003-0817LE

3. Marini JJ, Gattinoni L. Management of COVID-19 Respiratory Distress. JAMA. Published online April 24, 2020. doi:10.1001/jama.2020.6825

4. Solaimanzadeh I. Acetazolamide, Nifedipine and Phosphodiesterase Inhibitors: Rationale for Their Utilization as Adjunctive Countermeasures in the Treatment of Coronavirus Disease 2019 (COVID19). Cureus 2020;12(3): e7343. doi:10.7759/cureus.7343

5. Luks AM, Swenson ER. COVID-19 Lung Injury and High Altitude Pulmonary Edema: A False Equation with Dangerous Implications. Am J Respir Crit Care Med . 2020 Mar 30. doi: 10.1164/rccm.202003-0817LE. [Epub ahead of print].

6. Siddiqu HK, Mehra MR. COVID-19 Illness in Native and Immunosuppressed States: A ClinicalTherapeutic Staging Proposal. Journal of Heart and Lung Transplantation. Published online March 20, 2020. doi:10.1016/j.healun.2020.03.012

7. Zhu N, Zhang D, Wang W, et al. A novel coronavirus from patients with pneumonia in China. $N$ Engl J Med .2020;382(8):727-733. doi:10.1056/NEJMoa2001017

8. Li Y, Li H, Zhu S, et al. Prognostic Value of Right Ventricular Longitudinal Strain in Patients with COVID-19. JACC: Cardiovascular Imaging. Published online April 28, 2020. doi:10.1016/j.jcmg.2020.04.014

Figure 1. Suggested pathways for SARS-CoV-2 mediated ACE upregulation and ACE 2 downregulation. Endothelial activation pathways leading to intense pulmonary vasoconstriction in COVID-19: SARS-CoV-2 
spike protein binds to ACE 2 on the pulmonary epithelium and causes TACE-mediated shedding of ACE 2 ectodomain resulting in downregulation of ACE 2. Activated TACE causes concomitant shedding of soluble TNF $\alpha$ and IL-6 receptor on the apical, basal and basolateral side of the epithelial cell. TNF $\alpha$ causes increased expression of AT1R on endothelial cells. This results in increased AT II mediated harmful effects and reduced AT 1-7 mediated protective actions. High AT II activity reduces the expression of AMPK, a mediator for balance between ACE and ACE 2. AT II increase the conversion of TACE dimer to TACE monomer (active form) via AT1R/p38 MAPK and AT1R/ERK pathway. Thus, a vicious cycle of ACE/ACE 2 overactivity is established (Mechanistic pathways as shown in Inset). Due to relatively high expression of AT1R on pulmonary endothelium, vasoconstrictive actions of AT1R are only partly counterbalanced by AT2R mediated vasorelaxation. Increased levels ET-1 and peroxynitrite with relatively reduced levels of NO results in intense pulmonary vasoconstriction. (Pathways highlighted green are relatively activated while red highlights represent relative inactivation)

ACE: angiotensin-converting enzyme; ACE 2: angiotensin-converting enzyme 2; AT II: Angiotensin II; AT I: Angiotensin I; AT1R: Ang II type-1 receptor; AT2R: Ang II type-2 receptor; NOX: nicotinamide adenine dinucleotide phosphate oxidase; LOX-1: Lecithin-Like oxLDL Receptor-1; ETAR: Endothelin-1A receptor; B2R: B2 receptor; eNOS: endothelial nitric oxide synthase; SOD: superoxide dismutase; NO: nitric oxide; PLC: phospholipase C; PKC: protein kinase C; TACE: Tumor necrosis factor alpha converting enzyme; s-TNF- $\alpha$ : soluble tumor necrosis factor alpha; IL6R1: interleukin 6 receptor; IL6: interleukin 6; AT 17:angiotensin 1-7; PI3K/PKB/Akt: phosphoinositide-3-kinase-protein kinase B/Akt; AMPK: 5' adenosine monophosphate-activated protein kinase; miR: microRNA; EC: endothelial cell; VSMC: vascular smooth muscle cell.
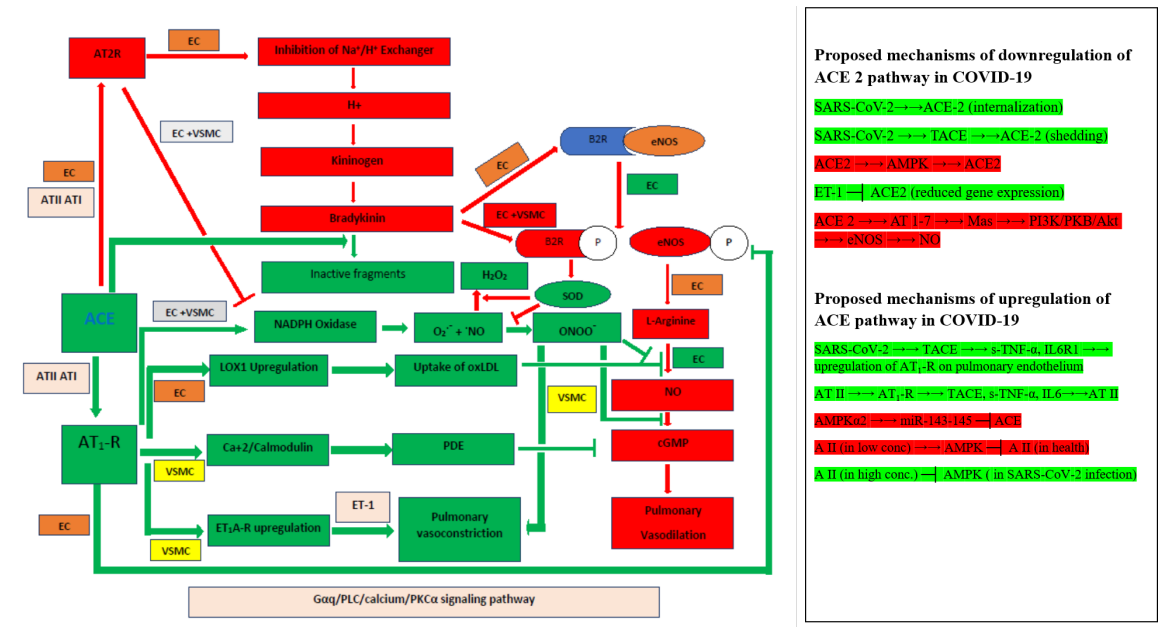